\title{
National Brain Tumor Foundation
}

National Cancer Institute

\section{Source}

National Cancer Institute. National Brain Tumor Foundation. NCI Thesaurus. Code C39465.

The National Brain Tumor Foundation (NBTF) is a national non-profit health organization dedicated to providing information and support for brain tumor patients, family members, and healthcare professionals, and supporting research efforts to develop new modalities of brain tumors treatment. 